\title{
Benefícios do ofurô na redução da dor em recém-nascidos pré- termo: uma revisão integrativa
}

Angela do Carmo Silva, ${ }^{1}$ Bianca Bueno Brito Silva, ${ }^{1}$ Rafaella Silvestre Borcades, ${ }^{1}$ Juliana Gomes de Lima Tostas, ${ }^{2}$ Leissa da Silva Melo-Figueiredo, ${ }^{2,3}$ Adalgisa Ieda Maiworm, ${ }^{2,4}$ Francisco José Salustiano da Silva, ${ }^{2,5}$ Laisa Liane Paineiras-Domingos ${ }^{2,6 *}$

\section{Resumo}

Introdução: O recém-nascido pré-termo (RNPT) é o bebê nascido após a interrupção da gestação antes de completar 37ạ semana gestacional. Diante de inúmeras consequências deste nascimento precoce, a sensação da dor é identificada com uma intensidade maior, pois suas vias inibitórias não estão totalmente desenvolvidas no RNPT. Muitos recursos têm sido sugeridos como promotores de bem-estar e consequente redução da dor na intervenção fisioterapêutica nos RNPT, como o ofurô. Objetivo: Esta revisão integrativa buscou identificar os benefícios do ofurô no bem estar do $\mathrm{RN}$ relacionado à dor. Metodologia: A presente revisão integrativa foi realizada através de referências bibliográficas atuais que abordam a saúde do RNPT e o ofurô, como uma técnica fisioterapêutica aquática capaz de promover o bem-estar e redução dor desses bebês. Conclusão: Por meio da literatura consultada, foi possível identificar que o ofurô é capaz de oferecer aos RNPT uma abordagem humanizada, considerada como uma das técnicas mais indicadas. Promove melhora adaptativa ao ambiente, alívio da dor, ganho de peso, diminuição da frequência cardíaca e da irritabilidade.

Descritores: Recém-nascido prematuro; Fisioterapia; Hidroterapia; Banhos; Imersão.

\section{Abstract \\ Benefits of the hot tub in reducing pain in preterm newborns: an integrative review}

Introduction: The preterm newborn (PTNB) is the baby born after the termination of pregnancy before completing 37 weeks of gestation. In view of the numerous consequences of this early birth, the sensation of pain is identified with greater intensity, as its inhibitory pathways are not fully developed in PTNB. Many resources have been suggested as promoters of well-being and consequent pain reduction in physical therapy interventions in PTNBs, such as the hot tub. Objective: This integrative review sought to identify the benefits of the hot tub on the well-being of newborns related to pain. Methodology: This integrative review was carried out using current bibliographic references that address the health of the PTNB and the hot tub, as an aquatic physiotherapy technique capable of promoting the well-being and reducing pain of these babies. Conclusion: Based on the consulted literature, it was possible to identify that the hot tub is able to offer PTNB a humanized approach, considered one of the most indicated techniques. It promotes an adaptive improvement to the environment,
1. Faculdade Bezerra de Araújo (FABA), Rio de Janeiro, RJ, Brasil.

2. Departamento de Fisioterapia, Faculdade Bezerra de Araújo (FABA), Rio de Janeiro, RJ, Brasil.

3. Hospital da Mulher Heloneida Studart (HMHS), São João de Meriti, RJ, Brasil.

4. Departamento de Fisioterapia, Universidade do Estado do Rio de Janeiro. Rio de Janeiro, RJ, Brasil. Janeiro, RJ, Brasil.

5. Associação Brasileira de Fisioterapia Integrativa e Práticas Integrativas e Complementares em Saúde (ABRASFIPICS), Rio de Janeiro, RJ, Brasil;

6. Laboratório de Vibrações Mecânicas e Práticas Integrativas (LAVIMPI), Departamento de Biofísica e Biometria e Policlínica Piquet Carneiro (PPC), Universidade de Estado do Rio de Janeiro (UERJ). Rio de Janeiro, RJ, Brasil.

*Endereço para correspondência:

Rua Carius, 179

Rio de Janeiro, RJ. CEP 23052-180.

E-mail: laisanit@gmail.com

BJHBS, Rio de Janeiro, 2020;19(1):63-68

Received on 03/30/2020. Approved on 06/08/2020.

pain relief, weight gain, decreased heart rate and irritability. Keywords: Infant; Physical therapy specialty; Hydrotherapy; Baths; Immersion.

\section{Resumen}

Beneficios de la bañera de hidromasaje para reducir el dolor en los recién nacidos prematuros: una revisión integradora

Introducción: El recién nacido prematuro (RNPT) es el bebé nacido después de la interrupción del embarazo antes de completar las 37 semanas de gestación. En vista de las numerosas consecuencias de este nacimiento prematuro, la sensación de dolor se identifica con mayor intensidad, ya que sus vías inhibitorias no están completamente desarrolladas en RNPT.Se han sugerido muchos recursos como promotores del bienestar y la consiguiente reducción del dolor en las intervenciones de fisioterapia en los RNPT, como la bañera de hidromasaje. Objetivo: Esta revisión integradora buscó identificar los beneficios de la bañera de hidromasaje en el bienestar de los recién nacidos 


\section{Artigo de revisão}

relacionados con el dolor. Metodología: Esta revisión integradora se realizó utilizando referencias bibliográficas actuales que abordan la salud del RNPT y la bañera de hidromasaje considerada una técnica de fisioterapia acuática capaz de promover el bienestar y reducir el dolor de estos bebés. Conclusión: A través de la literatura consultada, fue posible identificar que el jacuzzi

\section{Introdução}

De acordo com a Organização Mundial de Saúde, toda criança é considerada prematura antes de $37^{a}$ semanas de gestação, sendo chamado de recém-nascido prematuro ou pré-termo (RNPT). Quanto ao peso ao nascer, o RNPT poderá ser considerado de baixo peso, quando o peso de nascimento for inferior a $2500 \mathrm{~g}$, independentemente da idade gestacional".

Segundo a International Association for the Study of Pain, "a dor é definida como uma experiência sensorial e emocional desagradável", que geralmente são "associadas à lesão tecidual ou descrita em termos de tais lesões". Isto ocorre com os RNPT, como é preconizado pela fisiopatologia da nocicepção, dos neurotransmissores e da presença de receptores no sistema nervoso central (SNC) e periférico (SNP). ${ }^{2}$

Selestrin, 20113, acrescenta ainda que a dor é mais propriamente uma percepção, da mesma forma que a visão e a audição, do que simplesmente uma sensação. Ela envolve uma sensibilização das alterações químicas nos tecidos e uma posterior interpretação sobre se tais mudanças são ou não prejudiciais ao organismo, sendo processada em nível cortical. Essa percepção é real, tenha ou não ocorrido uma lesão, e a cognição está envolvida na formulação dessa percepção, havendo consequências emocionais e respostas comportamentais ante os aspectos cognitivos e emocionais da dor.

Segundo Albergaria e colaboradores, ${ }^{4}$ a dor tem a finalidade de fazer a proteção do corpo, fazendo com que o indivíduo reaja a esse estímulo doloroso. Apesar de ser um sintoma universal, não é expressa da mesma forma pelos indivíduos, principalmente pelos RN. Os estímulos e procedimentos dolorosos causam reações fisiológicas desagradáveis e o RN já possui a capacidade funcional e neuroquímica para a recepção, transmissão e integração desses estímulos por nociceptores, pelo desenvolvimento anatômico e fisiológico das vias de identificação da dor. ${ }^{5}$ A dor pode trazer consequências emocionais ou orgânicas, que podem comprometer o desenvolvimento do RN, principalmente o RNPT, pois os seus receptores sensoriais são extremamente sensíveis a estímulos externos. ${ }^{6}$ puede ofrecer un enfoque humanizado a RNPT, como una de las técnicas más indicadas. El promove una mejora adaptativa del medio ambiente, alivio del dolor, aumento de peso, disminución de la frecuencia cardíaca e irritabilidad.

Palabras clave: Recien nacido prematuro; Fisioterapia; Hidroterapia; Baños; Imersión.

Nas unidades de terapia intensiva neonatais (UTINs) são adotados diversos procedimentos de rotina que geram dor e desconforto para os pacientes, uma vez que em sua maioria não podem ser evitados. Cada RN internado recebe cerca de 50 a 150 procedimentos dolorosos por dia, como procedimentos fisioterapêuticos, tais como aspiração de vias áreas, manobras de reexpansão e higiene brônquica dentre outros procedimentos que podem ocasionar a piora das condições ventilatórias e hemodinâmicas do paciente5. O contato com os estímulos dolorosos desencadeia processos inflamatórios, ocasionando continuidade no ciclo de dor. O excesso desses estímulos aumenta a quantidade de oxigênio, podendo causar distúrbios de oxigenação no cérebro. 5

O RNPT sente mais dor do que os indivíduos de faixa etária mais velha, principalmente quando submetidos a estímulos dolorosos. Isso ocorre devido à percepção e inibição da dor. As fibras nociceptores no final do período fetal e no neonato são iguais à do adulto.? A mielinização incompleta ao nascimento não é a ausência de função, e sim, a velocidade da condução que nos neonatos, é compensada pela distância mais curta percorrida pelos potenciais de ação nos nervos periféricos. ${ }^{8}$ O circuito reflexo espinal funcional desenvolve-se quase que simultaneamente com o crescimento dos aferentes periféricos em direção à medula espinal. $\mathrm{Na} 24^{\mathrm{a}}$ semana de idade gestacional ou mais são identificados neurônios e vias nervosas suficientes para processar a sensação dolorosa no tronco encefálico. ${ }^{8}$ Para Gosling e colaboradores, ${ }^{9}$ episódios de dor ativam fisiologicamente a rede neuronal da neuromatriz da dor, o que causa modificações corticais relacionadas à dor.

Albergaria e colaboradores ${ }^{4}$ complementa que em pacientes pediátricos, muitas vezes há negligência pelos profissionais de saúde devido à dificuldade de comunicação nessa faixa etária, porém é sabido que os componentes neuroanatômicos, fisiológicos e neuroquímicos necessários à percepção da dor desenvolvem-se desde a vida intrauterina. Ao nascimento, esses processos não estão totalmente 
organizados, e a adaptação dependerá dentre outros fatores, das características do estímulo, do contexto e do estado comportamental no momento em que ocorre o estímulo doloroso.

Veras e Regueira ${ }^{2}$ sinalizam que a subjetividade do fenômeno doloroso dificulta a avaliação da intensidade, especialmente no neonato. Na avaliação do estímulo doloroso, autores recomendam indicadores fisiológicos como: a frequência cardíaca (FC); a frequência respiratória (FR); a variabilidade da $\mathrm{PCO}^{2}$; a sudorese palmar; o fluxo sanguíneo da pele; a pressão arterial (PA); a saturação de oxigênio (SaPO2) e a tensão transcutânea de oxigênio.

Não existe um instrumento completo para avaliação da dor em neonatos e/ou crianças. Por isso, os profissionais de saúde precisam ter conhecimento e entender os limites de cada ferramenta. Algumas escalas têm sido propostas em berçários para mensuração e acompanhamento da manifestação da dor como: o Sistema de codificação da atividade facial neonatal (NFCS), a Escala de avaliação de dor (NIPS) e o Escore para avaliação pós-operatório do RN - CRIES, propostas por Selestin, ${ }^{3}$ a Escala PIPP (perfil de dor dos recém-nascidos prematuro), proposta por Silva e colaboradores, ${ }^{10}$ e a Escala OPS (Escala de dor objetiva) descrita por Albergaria e colaboradores. ${ }^{4}$

Ultimamente, as intervenções terapêuticas no ambiente de UTIN têm sido sustentadas pela abordagem conhecida como humanização. Desde então, vários hospitais, predominantemente do setor público, começaram a desenvolver ações "humanizadoras" ${ }^{11}$ De acordo com Moreira e colaboradores, ${ }^{12}$ a humanização é o contato e/ou vínculo entre o profissional e o indivíduo alicerçado em ações guiadas pela compreensão e pela valorização dos sujeitos, reflexo de uma atitude ética e humana", ou seja, a qualidade do cuidado, que incluiria a valorização dos trabalhadores e o reconhecimento dos direitos dos usuários. A humanização é compreendida como: princípio de conduta de base humanista e ética; movimento contra violência institucional na área da saúde; política pública para atenção e gestão no SUS; metodologia auxiliar para gestão participativa, e Tecnologia do cuidado na assistência à saúde. ${ }^{11}$

O Programa de Humanização do Pré-Natal e do Nascimento (PHPN) garante à mulher dar à luz recebendo assistência humanizada e de qualidade e estabeleceu os princípios da atenção a ser prestada nos diferentes níveis de atenção pública.Para Cruz, Sumame Spíndola, ${ }^{13}$ os cuidados prestados ao RN imediatamente após o parto são essenciais para a adaptação do bebê, diminuindo a morbimortalidade neonatal. O delicado momento de transição do meio intra para o extrauterino é marcado por inúmeras mudanças para a criança. O meio intrauterino proporciona um ambiente de aconchego, de temperatura e luminosidade constantes, os ruídos são ouvidos suavemente, não necessitando de esforço para realizar as funções vitais. Com o nascimento o bebê vai se adaptando gradualmente ao meio extrauterino superando as dificuldades inerentes ao seu desenvolvimento.

De acordo com Gonçalves e colaboradores, ${ }^{14}$ nas UTINs, os RNPT estão sujeitos a um ambiente estressante e a muitas intervenções dolorosas. Contudo, algumas práticas têm sido adotadas para favorecer a assistência humanizada ao bebê e sua família, com medidas que reduzam o estresse, a dor, a manipulação excessiva e que permitam a interação entre o bebê e sua família.

Efeitos da água aquecida têm sido aproveitados para fundamentá-la como meio terapêutico para os RNPTs. ${ }^{14,15}$ Ribeiro e colaboradores ${ }^{5}$ defende o uso da água como terapia, capaz de tratar diversas enfermidades, pois a mesma, atua nos diversos sistemas do organismo, gerando benefícios e conforto aos pacientes e consequentemente a melhora da dor. Diante de inúmeros efeitos e benefícios, uma abordagem fisioterapêutica aquática humanizada sugerida para os RNPT é o ofurô, ${ }^{5}$ uma técnica que foi descrita por oferecer estabilidade nos sinais vitais e melhora no quadro álgico dos RNPT. ${ }^{14}$ Adicionalmente, oferece melhor resposta adaptativa ao ambiente, promovendo uma organização dos sistemas comportamentais, motores e fisiológicos. ${ }^{1}$

\section{Metodologia}

A presente revisão integrativa realizou uma busca de referências bibliográficas que abordaram a saúde do RNPT, considerando: (a) o ofurô, como uma técnica fisioterapêutica aquática; (b) abordagem intervencionista em RNPT e (c) que discutissem a promoção do bem-estar e a redução da dor nesses RNPT.

A busca foi feita em agosto de 2019, utilizando como descritores as palavras "fisioterapia" and "ofurô" and "bem-estar" and "dor". Foram considerados artigos publicados nos últimos 5 anos, na língua portuguesa, consultados na base de dados Scielo e Bireme. Dois investigadores diferentes fizeram as análises dos artigos desconsiderando abordagens que envolvessem outras modalidades terapêuticas e artigos publicados antes de 2014. 


\section{Artigo de revisão}

\section{Resultados}

Foram selecionados três artigos: Gonçalves e colaboradores, ${ }^{14}$ Ataíde e colaboradores ${ }^{15}$ e Silva e colaboradores. ${ }^{16}$ Numa análise crítica dos estudos incluídos, alguns aspectos relacionados ao objetivo proposto nesta revisão integrativa foram destacados:

\section{Sobre a conceituação da técnica}

Para Gonçalves e colaboradores,$^{14}$ o ofurô é uma modalidade terapêutica aquática que atua de forma assistencial humanizada, adaptada do banho de imersão. O ofurô é capaz de transmitir aos RNPTs uma sensação semelhante àquela do útero materno, fornecendo um momento prazeroso, de relaxamento e aconchego. Com isso, ajuda na redução do estresse, da dor, da perda de peso e do tempo de internação.

Ataíde e colaboradores ${ }^{15}$ descrevem ainda que o ofurô, apesar de ser ainda uma conduta de humanização da assistência realizada de maneira empírica, ganhou uma frequência rotineira nas unidades de cuidados neonatais brasileiras. Estes autores acrescentam ainda que a imersão de RNPT em água aquecida pode favorecer na redução do estresse, perda de peso, tempo de internação, favorecendo um melhor desfecho dos cuidados neonatais. Entretanto, eles alertam que o ofurô é diferente do banho de imersão, uma vez que seu objetivo principal é o relaxamento, através das propriedades da água aquecida sem envolver a higiene corporal.

\section{Sobre a execução da técnica}

Silva e colaboradores ${ }^{16}$ afirmam que a imersão do RNPT em água morna dentro de um balde deve ser até a altura dos ombros/clavículas, e sendo posteriormente, suave e passivamente mobilizado por meio de flutuação assistida. A abordagem terapêutica é executada, uma vez que o terapeuta usa a ação da força de empuxo do meio aquático para promover movimentação espontânea, propriocepção, organização postural e/ ou alongamento muscular, mobilização articular e movimentação rítmica do RNPT. Considera-se com isso que as propriedades físicas da água aliam-se às propriedades terapêuticas da aplicação do calor.

Ataíde e colaboradores ${ }^{15}$ abordam a importância do controle do ambiente, da luminosidade, da temperatura da água sendo mantida "entre 30 a 37 ${ }^{\circ} \mathrm{C} "$. O uso de termômetros, luvas, toalhas e balde com capacidade de 9 litros, são fundamentais para o procedimento. Relatam ainda sobre o preparo do RNPT com o enrolamento utilizando toalha-fralda, este "enrolamento é realizado até a altura do ombro do RNPT". Logo após o enrolamento o RN "é imerso lentamente no balde em postura verticalizada com os pés para baixo, de frente para o profissional, até que a água esteja na altura de seus ombros". Sendo posicionada uma das mãos na "região cervical e a outra na sacral, encaixando-o no fundo do balde". Por fim, tem a "retirada do RNPT do balde", que deve ser realizada com muita cautela e com duas pessoas no local. Para os autores, a fralda contentora deve ser cuidadosa e lentamente solta e retirada, ainda com o RN dentro do balde. ${ }^{15}$ Com muito cuidado, o RNPT é virado, apoiando o pescoço e a cabeça de maneira que ele permaneça de costas para o fisioterapeuta. Com uma das mãos apoiando a parte ventral do RNPT, fixando o pescoço, a região anterior do tórax e uma das axilas, e com a outra mão nas nádegas do RNPT, ele é suspenso e retirado do balde. A mãe ou cuidador auxilia o procedimento, colocando a toalha nas costas do bebê, para que ele seja retirado do balde. O RNPT, então, é novamente contido na postura flexora, para que fique aconchegado junto à mãe ou cuidador.

\section{Sobre cuidados na aplicação do ofurô}

Gonçalves e colaboradores ${ }^{14}$ determinam alguns critérios para a interrupção do ofurô como: (a) qualquer sinal de desconforto e/ou instabilidade clínica do RNPT; (b) careta; (c) tremores ou movimentos espasmódicos ou sacudidos; (d) irritabilidade; (d) hiperflexão do tronco e das extremidades; (e) cianose ou mudanças na coloração da pele; (f) vômitos; (g) espirros; (h) engasgos; (i) mudanças na respiração, alteração do padrão respiratório, respiração irregular e/ou choro acentuado; (j) evacuação durante a conduta. ${ }^{14}$

Sobre os benefícios relacionados ao bem-estar e à redução da dor

Gonçalves e colaboradores ${ }^{14}$ apontaram que a imersão em água aquecida juntamente com a contenção em padrão flexor parece trazer os seguintes benefícios como: (a) redução da desorganização neuropsicomotora; (b) minimização do estresse; (c) sensação de relaxamento; (d) melhora do desempenho neuromuscular e da organização motora; (e) favorecimento da capacidade de autorregulação e interação com o ambiente. Segundo esses autores, à medida que os bebês se desenvolvem, eles se tornam mais tolerantes a manuseios, intervenções e estimulações, demonstrando mais organização sensorial. 
Gonçalves e colaboradores ${ }^{14}$ descrevem, enfim, efeitos na redução da sensibilidade das terminações nervosas, levando à diminuição da dor; ao relaxamento muscular; à redução do estresse pela diminuição da dor e do relaxamento muscular, diminuindo os níveis de cortisol; à vasodilatação, promovendo o aumento da circulação periférica; ao aumento da capacidade vital; ao aumento do retorno venoso e à sensação de bemestar por simular o útero materno.

\section{Discussão}

Medeiros e Mascarenhas ${ }^{1}$ realizaram um estudo observacional, em 2009, com 35 recém-nascidos (RN) de ambos os sexos e com peso entre 1.000 e $2.499 \mathrm{~g}$. Concluíram que o ofurô proporcionou aos RNs melhor resposta adaptativa ao ambiente, organização dos sistemas comportamentais, motores e fisiológicos e melhor interação ao meio, contribuindo beneficamente para o seu desenvolvimento sadio.

Silva e colaboradores ${ }^{16}$ realizaram um estudo quantitativo, em 2016, com 50 RNPT de ambos os sexos, com peso mínimo de 1.500 gramas. Foi realizada a hidroterapia em um balde com capacidade para $18 \mathrm{~L}$, em duas sessões de 10 minutos em dias alternados. Os RNPTs foram posicionados na posição vertical, sendo imersos no balde até ao nível da água na altura das clavículas e a mão do fisioterapeuta posicionada entre a mandíbula e a região cérvico-occiptal, favorecendo sua flutuação e permitindo sua movimentação. Um termômetro infantil foi utilizado para monitorar a temperatura da água. Concluíram que o ofurô proporcionou aos RNPT um ganho significativo de peso corporal, diminuição da frequência cardíaca após a hidroterapia.

Ribeiro e colaboradores ${ }^{5}$ realizaram um estudo de caso em um RN com 3 meses de vida, nascido por parto cesáreo, com diversas complicações. Foi utilizado o ofurô em 5 sessões, 1 sessão por dia, com à temperatura da água em $36^{\circ} \mathrm{C}$ com durabilidade de 10 minutos cada sessão. Foram efetuados movimentos que estimularam a organização sensório-motora com exercícios em linha média. Considerando que o ofurô é um recurso que pode proporcionar estabilidade nos sinais vitais dos $\mathrm{RN}$, contribuindo para o bem-estar e relaxamento, estes autores observaram melhora em seu quadro álgico, na irritabilidade, e nas frequências cardíaca e respiratória.

Ataíde e colaboradores ${ }^{15}$ estabeleceram critérios de indicações e contraindicações para o ofurô aos RNPT. Como indicações, recomendaram o ofurô para sinais de estresse, para o ganho de peso entre 1.250 a 2500 $\mathrm{kg}$. Como contraindicações, eles descreveram que não foram encontradas contraindicações específicas, mas eles atentam para alguns cuidados gerais para a imersão em água aquecida, como: febre, ferida aberta e doença infecciosa.

Considerando a aplicação da técnica, metodologicamente, o banho no ofurô precisa seguir determinados procedimentos. Assim, Medeiros e Mascarenhas ${ }^{1}$ concordam com Silva e colaboradores, ${ }^{16}$ quando descrevem que o RNPT é imerso em água morna até o pescoço, sem exposição à corrente de ar e com contenção do padrão flexor através do enrolamento com toalha-fralda, evitando o estresse, a desorganização motora e o gasto energético e visando proporcionar relaxamento e prazer.

\section{Conclusão}

Com base na literatura levantada nesta revisão integrativa, o ofurô tem sido uma das técnicas mais indicadas como tratamento do RNPT. Ele é capaz de promover uma melhora adaptativa ao ambiente, o alívio da dor, o ganho de peso, a diminuição da frequência cardíaca e da irritabilidade.

\section{Referências}

1. Medeiros JS, Mascarenhas MFPT. Banho humanizado em recém-nascidos prematuros de baixo peso em uma enfermaria canguru. Rev. Ter. Ocup. 2010;21:53-8.

2. Veras ACR, Regueira MJS. Dor no recém-nascido. In: Manual de Neonatologia; Simões, A. 1. ed. Rio de Janeiro: Guanabara, 2002. p. 24-5.

3. Selestrin CC. Fisioterapia sob o contexto da dor na unidade de cuidados intensivos neonatal. In: Sarmento JV, Carvalho FA, Peixe AAF. Fisioterapia respiratória em pediatria e neonatalogia. 2. ed. São Paulo: Manole, 2011. p 309-11.

4. Albergaria TFS, Souza GF, Souza MLSB. Escala de dor e sua aplicação em unidades de tratamento intensivo pediátrica. In: Associação Brasileira de Fisioterapia Cardiorrespiratória e Fisioterapia em Terapia Intensiva; Martins JÁ, Nicolau CM, Andrade LB, organizadores. PROFISIO Programa de Atualização em Fisioterapia Pediátrica e Neonatal: Cardiorrespiratória e Terapia Intensiva: Ciclo 4. Porto Alegre: Artmed Panamericana; 2015;2:9-25.

5. Ribeiro LF, Xavier GN, Kairala AL et al. VII World Congress on Communication and Arts, 2015, Salvador. A utilização da terapia aquática como método de redução da dor em UTI neonatal (relato de caso). 2015;313-5.

6. Carneiro TLDP, Molina PD, Santos KSS, et al. Avaliação da dor em neonatos prematuros internados na unidade de terapia intensiva neonatal após a fisioterapia respiratória. Revista do Instituto de Ciências. 2016;34:220-3.

7. Veronez M, Corrêa DAM. A dor e o recém-nascido de risco: percepção dos profissionais de enfermagem. Revista Cogitare Enfermagem. 2010;15:264-5.

8. Okada M, Teixeira M J, Tengan SK, et al. Desenvolvimento do sistema nociceptivo e supressor da dor. Rev. Med. 2001;80:78-93.

9. Gosling AP, Suassuna PD, Nascimento LPR. Fisioterapia no 


\section{Artigo de revisão}

manejo da dor. In: Associação Brasileira de Fisioterapia Neurofuncional; Garcia CSNB, Facchinetti LD, organizadoras. PROFISIO Programa de Atualização em Fisioterapia Neurofuncional: Ciclo 2. Porto Alegre: Artmed Panamericana; 2015;2:37-49.

10. Silva YP, Gomez RS, Máximo TA, et al. Avaliação da dor em neonatologia. Revista Brasileira Anestesiol. 2007;57:565-74

11. Rios IC. Humanização: a essência da ação técnica e ética nas práticas de saúde. Rev Bras Educ Med. 2009;33:254.

12. Moreira MADM, Lustosa AM, Dutra F, et al. Políticas públicas de humanização: revisão integrativa da literatura. Ciênc Saúde Coletiva. 2015;20:3231-42.

13. Cruz DCS, Sumam NS, Spíndola T. Os cuidados imediatos prestados ao recém-nascido e a promoção do vínculo mãebebê. Rev Esc Enferm USP. 2007;41:690-7.
14. Gonçalves RL, Carvalho MGS, Sanchez FF, et al. Hidroterapia com ofurô como modalidade de fisioterapia no contexto hospitalar humanizado em neonatologia. In: Associação Brasileira de Fisioterapia Cardiorrespiratória e Fisioterapia em Terapia Intensiva; Martins JA, Andrade LB, Ribeiro SNS, organizadoras. PROFISIO Programa de Atualização em Fisioterapia Pediátrica e Neonatal: Cardiorrespiratória e Terapia Intensiva: Ciclo 6. Porto Alegre: Artmed Panamericana. 2017;1:59-90.

15. Ataíde VP, Barbosa JSV, Carvalho MGS, et al. Ofurô em recém-nascidos pré-termo de baixo peso: relato de experiência. ASSOBRAFIR Ciência. 2016;7:14-20.

16. Silva HA, Silva KC, Reco MON, et al. Efeitos fisiológicos da hidroterapia em balde em recém-nascidos prematuros. Rev Ter Ocup Univ. 2017;28:3009-15. 